\title{
RMC: Real-Time Monitoring and Controlling Approach on Networks
}

\author{
G. Naveen Samuel ${ }^{1}$, Immanuel Ganadurai ${ }^{2}$ \\ ${ }^{1}$ (Computer Science and Engineering, Joe Suresh Engineering College, India) \\ ${ }^{2}$ (Computer Science and Engineering, Karpagam University, India)
}

\begin{abstract}
A GUI based architecture for monitoring the operations and controlling the activities of the client machines placed in a network by an approach named RMC (Real-time Monitoring and Controlling) system. A client - server approach of the net spy allows the administrator to view those client systems connected to the LAN. The network administrator chooses needed clients with the help of their IP address for the monitoring process that enhances various events as system information, memory usage, desktop capturing and the tasks that are currently running under those clients. It captures the resources and each operation made in the client secretly. Here we enroll another process of controlling those monitored resources. The control utility includes controlling hardware like keyboard, mouse and also locking the client's machine. It performs shutdown operations on the clients from the server. Combination of the controlling aspect along the monitoring process includes a cost efficient approach in maintaining a reliable network.
\end{abstract}

Keywords: API interface, GUI interface, screen capture, Net spy and RMC approach.

\section{Introduction}

Real-time Monitoring System (RM) is GUI based software for monitoring the clients in a network. Net Spy enables an administrator to view the number of client systems connected to a LAN. Client Systems are monitored through their IP address. While a client enters a network, it is enhanced with a name and an IP address to be registered in the server. Monitoring is efficient in all organizations for maintaining records about the clients entering and exiting their network. Various events are monitored such as system information, usage of memory, capturing desktop activities and tasks running under clients. System Information contains OS name and version, processor detail, hard disk information and RAM details [3]. Monitoring a client can be a security operation prevailed over a network to keep a reliable flow of information between client and server. Clients may enroll their IP with the server for communication purpose but administrator takes rights on clients to make a security service. RM ensures a current state monitoring system which provides a facility of maintaining actions of the clients within the network circle. Clients may work against the organization and make them to collapse the goal they fixed for the yearly needs. But RM systems are used in their organization to make them secure by monitoring those clients and make their motto to be diminished. Most of the monitoring systems ensure traffic monitoring by the use of packet analyzer [12]. Operations can be monitored only through the IP addresses that are provided by the clients.

Real-time Controlling system $(R C)$ is an approach engaged only on controlling the clients from the server. The server or network administrator in the network controls each activity of their clients enrolled in their network. Controlling system is also made using the IP address of the clients. An organization controlled by a head, controls all the operations of their employees or clients by giving different rights for each clients. This is organized also in networks by enabling some rights for each client $[5,17]$. Controlling software enables the network administrator to take control of their clients from their server monitor. Administrator enhances several rights along the clients for security purposes and maintains the network by provided commands over the clients to be performed by the clients to be constant in the network circle. Real-time controlling includes the control utility such as controlling hardware support of for the clients and locking their machines if not needed. It also performs shut operations on the clients from the server. Enabling this controlling system can make a cost efficient process for the server to work through their network. RC enabled networks can be a profitable approach for the organizations to work consistently. Servers enable their operation by commanding their clients to proceed with their work along server's interaction on them [18, 23]. Networking enables those clients that are engaged along the server to process a schematic referenced structure of data transferring options. Controlling state of a system is the value added process of the system with an augmented security over a network environment. 


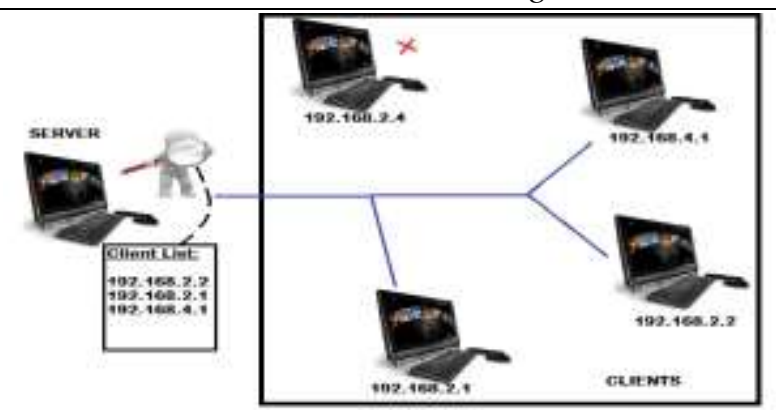

Fig. RMC Approach

Real-time Monitoring and Controlling (RMC) approach is enhanced with both these operations in a single process that can be entitled with the network procedures. Usage of this approach on a network will power the system with secure formulae. This approach will process along the performance of the clients and server to interact with the processing style of the network. Administrator takes full responsibility of the network by working with both monitoring and controlling operation that is engaged in an organization to fulfill their goal. Maintenance of the network is enabled with this approach to stimulate a proper security structure of a network by propagating through the communication process [4].

\section{Related Works}

In present scenarios, there are softwares performing monitoring options on the network. These monitoring approach are implemented in several systems for traffic management. Softwares that can perform both monitoring and controlling operations are no more present in this existing environment. It is difficult to control the system only with the monitoring phase. Servers or network administrators are enhanced only with monitoring the operations made by the clients and if needed to control them, they need to make a separate manual process that means reaching them to control their activities. It requires more efforts and also its cost expensive. It may need several softwares in engaging both the operations in a network. Manual procedures may lead to several failures in control [14]. For enabling an efficient RMC (Real-time Monitoring and Controlling) system, we introduce a procedure for making both the operations by the server. This approach includes both monitoring as well as controlling the activities made by the clients. Organisations working with RMC can be familiar in securing a constant network connection. A network administrator can work easily with this approach by organising the network connection in a single software approach proposed [21]. Enabling RMC reduces extra burden of purchasing two or more softwares for monitoring and controlling the network. Here server can identify a network traffic easily by monitoring each clients working in a network and can control them with some system commands. GUI based architecture is also used to enhance a live stream of capturing the activities done in the desktops of the clients present in a network [7,16]. This GUI architecture is enabled for a real-time approach of the RMC system.

a) Monitoring Phase:

III. RMC Approach

Server lists the clients using their IP address connected to the LAN. The API program is linked to all the files in the operating system. API is an interface between the user and the software components used in the system. It makes the remote procedure call method to interact with the operating system. IP addresses of the clients interacting along the network are listed along the server state [1,22]. Those clients needed to interact along the network are made to register their IP address and their name on the list provided by the server. Client's IP address is used for monitoring them by calling API functions of the client. Server enhances this operation and checks or monitors the required details of the clients and makes the GUI interface to enable a real-time monitoring to the server $[8,19]$. GUI interface makes a graphical representation of the client's current working particulars and furnishes those activities that are made in it.

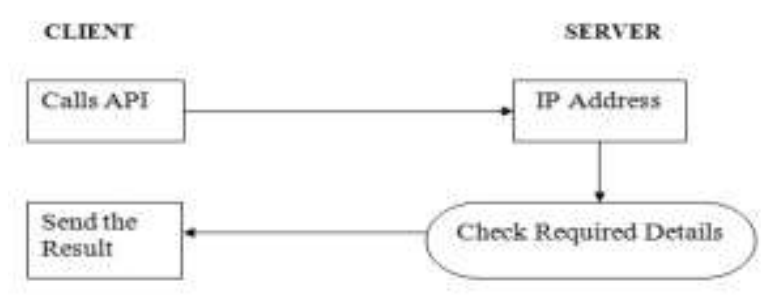

Fig. Monitoring Phase 


\section{b) Controlling Phase:}

This phase is devised as the second phase of this approach that is proposed to take control over the clients connected onto a network. Here the monitored state needs a result of action on the client to make its state of action excellent with the help of this phase [6]. API interface plays the main role in this phase by regulating the commands that are organized by the server or the administrator in controlling the clients operations. Administrator or the server organizing the network make the commands of controlling power over the client engaged with them $[2,11]$. Forcing the clients to make the work give by the organizer or the server and work according to the commands entitled by them is the main task prevailed in this phase.

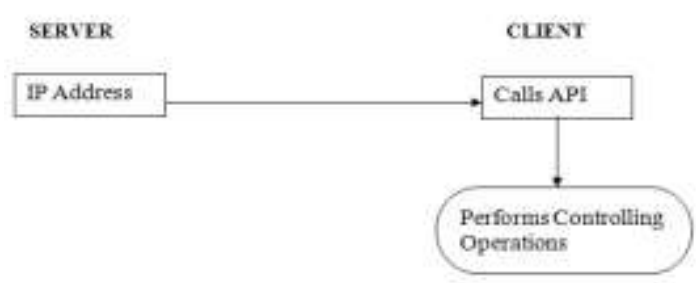

Fig. Controlling Phase

The server selects an IP address of the client and makes a call for API programming commands to the selected client. Client then makes the required operation of the API for computing the task [13]. Thus it performs along it for making control operations on the system. Client is enhanced with a regulated operation to control its state of work or the traffic engaged by it.

\section{Implementation of RMC}

RMC is based on GUI software interface which helps in making a live monitoring of the clients operations. RMC approach consists of two different modules with the Net Spy operation such that Monitoring and Controlling module. Here we structure a new net spy device by enrolling both these modules into it, while each performs its own task. Both modules make an effective procedure in maintaining constant achievements in the organisation. Operations are enhanced according to the goal entitled by the organiser of the network. Clients participating in a network are organised with their IP address listed in the server for regulating a systematic process of evaluating a solution to the improper work issue of the clients in the field [15, 22]. RMC approach is proposed for achieving those requirements of an organisation working on temporal changes made across a network. Implementing RMC is basically advised with the administrator's rights prescribed over a network for performing an efficient task that surrounds the contributions of the clients ensuring the same network. Client maintains a consistent approach with the server to retain the tasks measured by the organisation which is controlled by the administrator of the system. Attributes of the clients are also monitored then and there with this approach and controls every actions organised by them to compromises the procedure enforced by the administrator or the organiser.

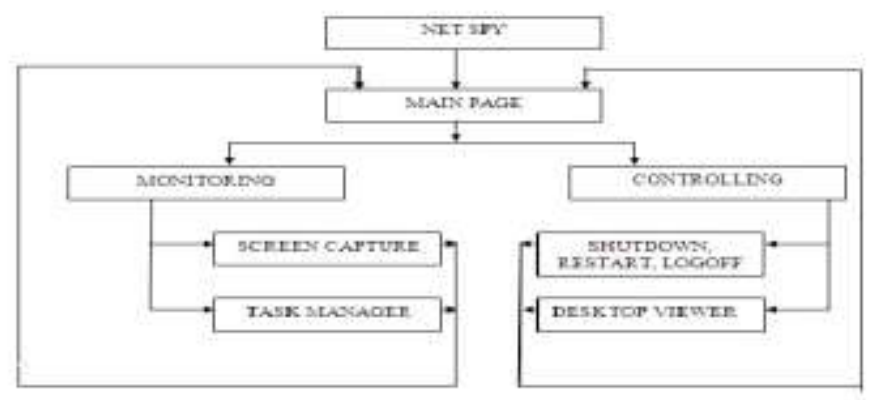

Fig. RMC Approach

\section{a) Monitoring:}

Clients are monitored along the network to make a secure working strategy. Server makes use of this module that performs operations as Screen Capture and Task Manager.

Screen Capture: Here the live screen is telecasted from the clients to the server. The current screen is captured from the client system and is displayed in the server. The administrator or the server enhances an efficient watch over the user who works in the system currently. Here the server selects the IP address of the client from the client list and makes a call to the specified client with an API program. Also it selects the 
operation type of the client as a string message that consists of the required operations to be made by the client. The client then receives the message and it makes the required solution for the server. The client first checks whether the IP call is same as its IP address. Secondly, it works for the server by capturing the screen and it converts the screen into a bitmap and it converts the bitmap then into bytes. Here the stream of bytes is transferred from the client to the server. Server receives the stream and converts it into a bitmap image and the bitmap is displayed on the server as the output of the screen capturing operation.

Task Manager: Task manager lists all the processes that are running in the client system, including the background process. In screen, capture the user can easily cheat the administrator by minimizing the window and hiding the taskbar. But the task manager that is abstracted will display all the running process even though it is in the hidden state. User may activate several processes at a time that are organized in the task manager for ensuring the performance of the current system. Here it makes a specific process to be abandoned which is working with errors and may not respond to the system. Administrator can view the processes of the client used and also it can perform the required action of the server against the client's performance.

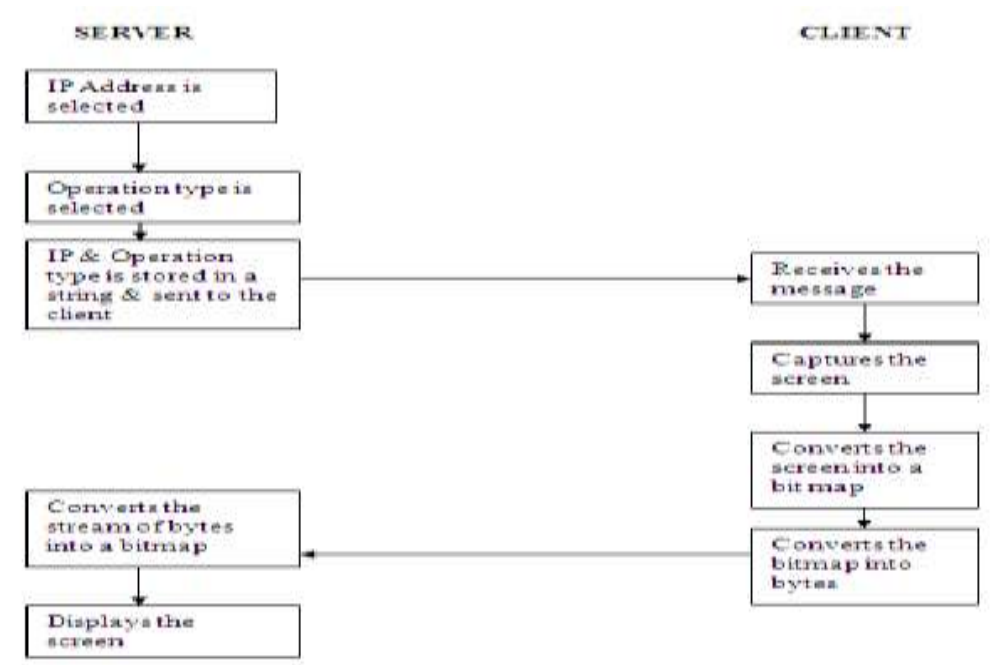

Fig. Screen Capture

\section{b) Controlling:}

Controlling module is entitled as the important modules of the RMC approach. Here different operations are enabled as desktop viewing and performing operations on the displayed information. Controlling involves operations as termination, shutdown, logoff or restart operations and so on. Each operation may be organized by the administrator or server for maintaining the performance of the network used by several clients. Here operations are performed specifically on a client by introducing its IP address on the server message. Server selects the IP address of the client which needs the server operation. Here the operation type is selected and it is enabled through the IP that is already been selected. IP address and the operation type selected by the server is organized as a string message that is sent to the client for enabling its required action. The client receives the message from the client, analyses it and performs the needed operations on it. Operations are performed by the API interface present in each client system. The message sent by the server first contacts the client API interface and makes the calls for enabling several operations on the client system.

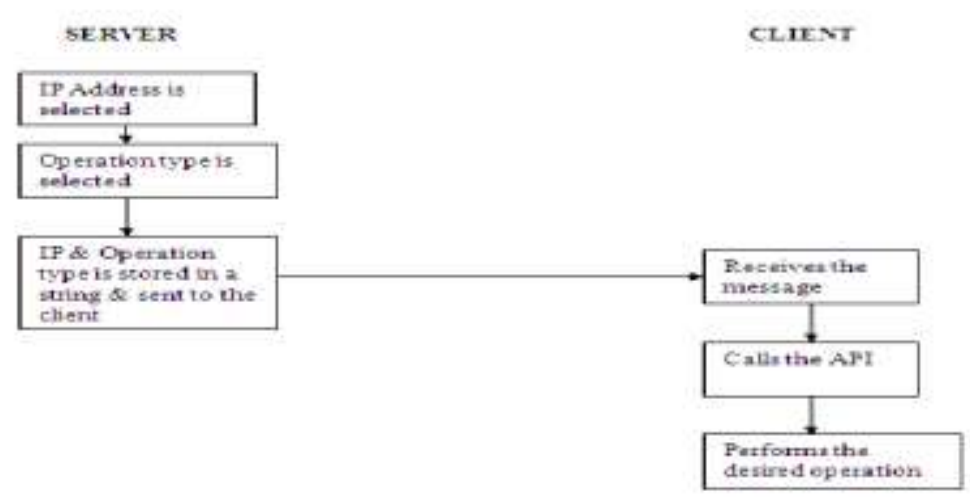

Fig. Performs Controlling Operations 


\section{Need of RMC Approach}

Most organizations need an eye on their employees work to fulfill their goals. Thus RMC approach is used here for making a real-time monitoring of their employees and makes them work properly by controlling them without any physical access. RMC plays the main role in capturing the employee's screen and displaying it in the administrator's system. Administrator also allows the employees present in the list to be logged into their network. RMC helps in terminating the programs that are running improperly in their client system. Organizations goals are to be succeeded with a necessary procedural works by the employees but if it is out of service in monitor and controlled by the administrator acting as a server [10]. Employee's system could be turned on only when it is registered in the employee's list at the time of connection orientation. Without the presence of the administrator in an organization, systems are not allowed to be accessed by the clients. They work on their organization and make a disciplined way of communication between their employees. If any operation to be aborted by the client, then the server makes a message to the client to withhold those works and work along the organization [6]. If the received message is rejected by the client, the server takes charge on the client by terminating their works with the help of RMC approach. Nowadays various educational institutions works with RMC approach to favor an excellent education to the students they handle. Education is organized through a network where movements of every student are watched periodically. Students are allowed to make their listening and writing works through the network based education system that are monitored then and there to show a good result in their performance in examinations. Monitoring each and every student to make them study well and construct their future with a disciplined environmental state of operations within them $[9,20]$. Teachers in the institutions acts as the administrator and takes charge of their students activities and make a perfect atmosphere over the institution. RMC approach is also used in many applications for favoring a reliable state of networking state [7]. RMC approach has secured a reliable and cost efficient software approach over the networking field.

\section{Performance Analysis}

Achieving a well disciplined and a profitable growth in an organization is not an easy task of the organizer. Employee's attitude of work should be analyzed at every moment to achieve an affordable goal. Organizers or the administrator must monitor every moments of their employee or client present in their organization or network. Monitoring alone could not show an efficient result in the organization. With the help of monitoring aspects, controlling of each activity of the clients is to be enhanced in this state [15]. Servers are not in a safe mode operation such as the clients working in their network can make some illegal source of operation against the network. Here the objective of the organization now is set to be with both monitoring each activities that are made currently and control them from the organizer's desk and not with their physical access to each employees working in their organization. This objective is achieved through the RMC approach enhanced in this paper and makes the organizer to be up-to-date about their employees working details. Persistence of the employees over the organizational needs is made to be in a procedure to fulfill or achieve their goals entitled with them $[9,13]$. While comparing to the present software's which are organized only for monitoring but not present their ideas in controlling the unwanted actions of the clients in the network. RMC approach makes a reliable system of monitoring all the current state of each system connected to the network and present an interactive control measures on them which could be engaged with any operation that are persisting in the system. While analyzing its performance with the organization which is in need of this approach, it can be measured to be perfect on their action persisting with this approach that maintains an affordable result. This research study with RMC throws light on the extensive use of statistical techniques for analyzing the reliable performance of the organizations [21]. The paper also furnishes a summary of the requirements in each organization and concludes with an efficient approach. It fulfils all the needs of the organizers for organizing their employees. A sensitive study is made along this paper to make the organization full interactive with this approach.

\section{Conclusion}

Finally coming to the conclusion of this paper a reliable RMC approach is devised and verified successfully. Real-time Monitoring and Controlling system has been designed and developed to the satisfaction of end users. Approach is built with a real-time strategic limit that could render both monitoring and controlling operations on the clients connected to the network. Administrator needs are satisfied along the specific attitude of the clients making their works against the network to establish a negative statics on it. Administrator makes use of this approach every time when the clients enter the network at a specific limit. Lists are maintained periodically at the server and the connections are established along the response given by the server or administrator. The system has been built with the latest technology to make the best of all resources. This approach is used specifically in education system to render a perfect study to the students and make them to engage with the teachers in a friendly atmosphere. It is also used probably in the organisations which consists of employees and works in a network structure to formulate a worthy achievement which regards the organiser's 
goal. Here the structural representation of the approach is statistically used all over the system to compromise a specific work needed for encrypting a system along the basic situations maintained by the organiser. RMC approach is user friendly as it shows a fast response through the administrator. It is simple in working throughout the statistical operations made by the clients in the system and it is spied by the organiser or administrator who is responsible for the entire work. It is also much interactive with clients and server present in the network which formulates an affordable approach to the administrator. RMC approach works easier than other systems in the networking environment. Future world is fully based on networking and monitoring of the structured networks which becomes an important task. With this approach specified in the paper, we could manage any remote system i.e. we can kill a process, shutdown, reboot and also lock a remote system. Several enhancements can be made along this paper in future to entitle this software approach to be reliable in the networking market.

\section{References}

[1] Chang Ho Choi , CSMonitor: a visual client/server monitor for CORBA-based distributed applications, Software Engineering Conference, 1998. Proceedings, Pg: 338-345.

[2] Dorsey, Robert E, 1992, The Voluntary Contributions Mechanism with Real Time Revisions, Public Choice, Springer, vol. 73(3), pages 261-82, April.

[3] Cary Deck \& Nikos Nikiforakis, "Perfect and Imperfect Real-Time Monitoring in a Minimum-Effort Game", Working Papers 1018, 2010,Chapman University, Economic Science Institute.

[4] V. Kapsalis, A. Kalogeras, K. Charatsis, G. Papadopoulos, "Seamless Integration of Distributed Real Time Monitoring and Control Applications Utilising Emerging Technologies", IECON'01: The 27th Annual Conference of the IEEE Industrial Electronics Society, Pg: 176-181, 2001 IEEE

[5] G. Chen, M. Neville, Q. Kong, "Distributed Network Management Using CORBA/TMN", Proceedings of the 7th IFIP/IEEE International Workshop on Distributed Systems Operation and Management (DSOM), 1996.

[6] J. Boyle, H. Truong, N. Nour, "Providing a web-based view of your managed network", Proceedings of 1997 IEEE International Conference on Communications (ICC'97), Montreal, Canada, June, 1997.

[7] Tony White, Andrzej Bieszczad, "The Expert Advisor:An Expert System for Real Time Network Monitoring", Northern Telecom

[8] [Rabie et al 1988] Rabie S., Rau-Chaplin A., Shibahara T., DAD: A Real-Time Expert System for Monitoring Data Packet Networks, IEEE Networks Magazine September, 1988.

[9] [White, Bieszczad 1992] White T.,Bieszczad A., A Customization environment for the Expert Advisor Network Management system, Canadian Conference on Artificial Intelligence, Vancouver, 1992.

[10] [Laffey, et al 1988] Laffey T., WeitzenKamp S., Read J., Kao S., Schmidt J., Intelligent Real-Time Monitoring, Proceedings AAAI 1988 pages $72-76$.

[11] Girish Revadigar, Chitra Javali, "Real Time Monitoring And Control Of Wireless Networks", International Journal Of Embedded Systems And Applications (Ijesa) Vol.2, No.1, March 2012.

[12] Ahmed N. Abdalla, Muhammad Nubli, Tan Chien Siong, Fauzan Khairi, A. Noraziah, "Enhancement of real-time multi-patient monitoring system based on wireless sensor networks", International Journal of Physical Sciences Vol. 6(4), pp. 664-670, 18 February, 2011.

[13] Rolf Stadler, Mads Dam, Alberto Gonzalez, Fetahi Wuhib, "Decentralized Real-time Monitoring of Network-wide Aggregates", Ladis 2008 proceedings.

[14] Lasse Klingbeil, TimWark, "AWireless Sensor Network for Real-time Indoor Localisation and Motion Monitoring “, 2008 International Conference on Information Processing in Sensor Networks.

[15] A. Witayangkurn A, *, M. Nagai B, K. Honda C, M. Dailey C, R. Shibasaki D, "Real-Time Monitoring System Using Unmanned Aerial Vehicle Integrated With Sensor Observation Service " International Archives Of The Photogrammetry, Remote Sensing And Spatial Information Sciences, Vol. Xxxviii-1/C22 Uav-G 2011, Conference On Unmanned Aerial Vehicle In Geomatics, Zurich, Switzerland

[16] W. Jiang, H. Schulzrinne, Modeling of packet loss and delay and their effect on real-time multimedia service quality, In Proc. NOSSDAV, June 2000.

[17] K. Stuhlmuller, N. Farber, M. Link and B. Girod, Analysis of video transmission over lossy channels, IEEE J. Select. Areas Commun., June 2000.

[18] A. R. Reibman, V. Vaishampayan, Y. Sermadevi, " Quality monitoring of video over a packet network. IEEE Trans.Multimedia, April 2004.

[19] Schill, Alexander, "DCE-The OSF Distributed Computing Environment Client/Server Model and Beyond", 283.International DCE Workshop. Karlsruhe, Germany, October 7-8, 1993. Berlin, Germany: Springer-Verlag, 1993.

[20] G. Chen, M. Neville, Q. Kong, "Distributed Network Management Using CORBA/TMN", Proceedings of the 7th IFIP/IEEE International Workshop on Distributed Systems Operation and Management (DSOM), 1996.

[21] L. Deri, B. Ban, "Static vs. Dynamic CMIP/SNMP Network Management Using CORBA", IBM Research Report, IBM Zurich Research Laboratory, 1996.

[22] "Wireless Medium Access Control (MAC) and Physical Layer (PHY) Specifications for Low-Rate Wireless Personal Area Networks (LR-WPANs)”, Institute of Electrical and Electronics Engineers, IEEE 802.15.4, 2003.

[23] N. Bulusu, J. Heidemann and D. Estrin, "GPS-less Low Cost Outdoor Localization For Very Small Devices", IEEE Personal Communications, Special Issue on Smart Spaces and Environments, Vol. 7, No. 5, October 2000, pp 28-34. 